\title{
Pulse consumption in Canadian adults influences nutrient intakes
}

\author{
Adriana N. Mudryj ${ }^{1}$, Nancy $\mathrm{Yu}^{2}$, Terryl J. Hartman ${ }^{3}$, Diane C. Mitchell ${ }^{3}$, Frank R. Lawrence ${ }^{3}$ \\ and Harold M. Aukema ${ }^{1 *}$ \\ ${ }^{1}$ Human Nutritional Sciences, University of Manitoba, Winnipeg, MB, Canada R3T 2N2 \\ ${ }^{2}$ Community Health Sciences, University of Manitoba, Winnipeg, MB, Canada R3T 2N2 \\ ${ }^{3}$ Nutritional Sciences, Pennsylvania State University, University Park, PA 16802, USA
}

(Submitted 27 July 2011 - Accepted 13 September 2011)

\section{Abstract}

Pulses (dry beans, peas, lentils) are nutrient-dense foods that are recommended as good choices in either the vegetable or meat and alternative food groups in Canada's Food Guide. To examine the prevalence and the effect of pulse consumption on nutrient intake in Canadian adults ( $\geq 19$ years), we analysed cross-sectional data ( $n$ 20156) from the 2004 Canadian Community Health Survey, Cycle 2.2. Participants were divided into non-consumers and quartiles of pulse intake. Sample weights were applied and logistic regression analysis was used to explore the association of nutrient intakes and pulse consumption, with cultural background, sex, age and economic status included as covariates. On any given day, $13 \%$ of Canadians consume pulses, with the highest consumption in the Asian population. The pulse intake of consumers in the highest quartile was 294 (SE 40) g/d and, compared with non-consumers, these individuals had higher intakes of carbohydrate, fibre and protein. As well, the micronutrient intake of pulse consumers was enhanced, resulting in fewer individuals who were below the estimated average requirement for thiamin, vitamin $\mathrm{B}_{6}$, folate, Fe, Mg, $\mathrm{P}$ and $\mathrm{Zn}$, compared with non-consumers. Although pulses are generally low in $\mathrm{Na}$, its intake also was higher in pulse consumers. Among the higher quartiles of pulse consumers, fruit and vegetable intake was one serving higher. These data indicate that pulse consumption supports dietary advice that pulses be included in healthful diets. Further studies elucidating the sources of increased Na in pulse consumers will be necessary so that dietary advice to increase consumption of pulses will maximise their nutritional benefits.

\section{Key words: Pulses: Adults: Nutrient intakes: Canadian Community Health Survey}

Pulses have been consumed for at least 10000 years and are among the most extensively used foods in the world. Nutritionally, they are characterised by high protein content (about 20-30\%), a very high proportion of carbohydrate (about 50-65\%) and a very low fat content (about 1\%). They are a significant source of many nutrients, including fibre, protein and $\mathrm{Fe}$, as well as many vitamins. However, their consumption in the Western world remains quite low at less than $3.5 \mathrm{~kg} /$ capita per year. In other parts of the world, annual pulse consumption can range from $10 \mathrm{~kg} /$ capita (South America and India) to $40 \mathrm{~kg} / \mathrm{capita}$ (Burundi) ${ }^{(1,2)}$.

Pulses are the edible seeds of members of the Fabaceae (Leguminosae) family. According to the FAO, pulse crops refer to crops harvested exclusively for their grain, including dry beans, peas and lentils. As defined further by the FAO, the definition excludes crops used for oil extraction, such as soyabeans and groundnuts or those harvested green for food, such as green beans and green peas ${ }^{(3-6)}$. For the purpose of the present study, the FAO definition is used and the term 'pulses' refers to the dry, edible variety of beans, peas and lentils, and excludes soyabeans, fresh beans and fresh peas.

Research supporting pulse consumption coupled with diets low in animal protein and high in grains and cereals has been substantial. Results from six clinical trials reported at the 2008 Pulse Crop Symposium held in Toronto showed that eating beans, peas, lentils and chickpeas can help combat chronic diseases such as heart disease, obesity and diabetes and contribute to overall good health. These clinical trials linked pulse consumption with a reduction in health problems such as obesity, diabetes and $\mathrm{CVD}^{(7-9)}$. Pulses contain a mixture of soluble and insoluble fibre, which lowers total serum and LDL-cholesterol and aids in gastrointestinal function, respectively ${ }^{(6,9)}$. With respect to cancer, the US Food and Drug Administration, Canadian Cancer Society and the World Cancer Research Fund also recommend the consumption of

Abbreviations: CCHS $2 \cdot 2$, Canadian Community Health Survey, Cycle $2 \cdot 2$; EAR, estimated average requirement; NHANES, National Health and Nutrition Examination Survey.

*Corresponding author: Dr H. M. Aukema, fax +1 204237 4018; email aukema@umanitoba.ca 
pulses to reduce cancer risk ${ }^{(10-12)}$. Nutritive components of pulses such as Se, Zn, fibre and folate, as well as phytochemicals such as saponins, protease inhibitors and phytic acid also may be associated with their anti-carcinogenic benefits ${ }^{(6,13)}$. A high consumption of pulses also is one of the eight components of the highly lauded Mediterranean diet ${ }^{(14)}$. Research has shown that their consumption leads to increased satiety, and high-fibre foods take longer to eat, increasing one's feeling of satiety ${ }^{(15)}$. While it remains inconclusive, studies have shown that pulse consumers had lower body weights and reduced waist circumferences compared with non-consumers ${ }^{(16)}$, making pulses ideal for individuals interested in weight $\operatorname{loss}^{(6)}$. In addition to being low in fat and $\mathrm{Na}$, pulses have a low glycaemic index, which may be a factor in diabetes prevention and management ${ }^{(6,17,18)}$. They do not contain cholesterol or gluten, and research has also examined their protective effects as prebiotics ${ }^{(8)}$.

Canada's Food Guide recommends the consumption of pulses as a good choice and considers a half-cup serving of pulses equal to one serving of vegetables, and a three-quarter-cup serving equal to one meat or alternative serving ${ }^{(19)}$. The advisory committee on the Dietary Guidelines for Americans in 2010 suggested shifting food intake patterns to include cooked dry beans and peas, while the US Department of Agriculture recommends that Americans consume 2.5 to 3.5 cups of pulses per week ${ }^{(20,21)}$. Data from the US National Health and Nutrition Examination Survey (NHANES) 1999-2000 showed that American adults consume one-third or less than the recommended serving of pulses. In our recent analysis of pulse consumption in the USA using the NHANES data we found that the average consumption of pulses was less than one cup per week. We also found that pulse consumption resulted in higher intakes of fibre, carbohydrate, protein, $\mathrm{Ca}, \mathrm{K}$, folate, $\mathrm{Zn}, \mathrm{Fe}$ and $\mathrm{Mg}$, with lower intakes of saturated as well as total fat ${ }^{(22)}$.

The purpose of the present study was to examine the demographics of the average Canadian pulse consumer using data from the Canadian Community Health Survey, Cycle $2 \cdot 2$ (CCHS 2.2). We report the prevalence of pulse intake in the Canadian population and assess the relationship between pulse consumption and nutrient intakes and diet quality.

\section{Experimental methods}

Data from the CCHS $2 \cdot 2$ conducted by Statistics Canada were used for this analysis ${ }^{(23)}$. This cross-sectional survey targeted respondents from all age groups living in private occupied dwellings in the ten provinces (Indian reserve occupants, residents of the territories of Nunavut, Yukon and the Northwest Territories, individuals residing in institutions and members of the Canadian Forces were excluded). Data collection began in January 2004 and continued throughout the year to remove seasonal effects. The main objectives were to gather information on the nutritional status of Canadians, and estimate the distribution of usual dietary intake in terms of foods, food groups, dietary supplements, nutrients and eating patterns among a representative sample of Canadians at national and provincial levels using a $24 \mathrm{~h}$ dietary recall. A grand total of 35107 adults and children completed the initial $24 \mathrm{~h}$ dietary recall. Following this, a subsample of 10786 completed a secondary recall $3-10 \mathrm{~d}$ later. The $24 \mathrm{~h}$ dietary recalls were collected primarily by face-to-face interviews by trained interviewers $^{(24,25)}$. Further details on the methods used in the CCHS $2 \cdot 2$ are available on the Statistics Canada Website ${ }^{(26)}$. Data for the present analysis were limited to adults aged $\geq 19$ years ( $n$ 20156) and to 1 d dietary intakes only. Respondents who did not consume any food or whose recalls were considered to be unreliable according to Health Canada were removed. Pregnant and breast-feeding women were included in the present study and although vitamin and mineral supplementation was coded for in the CCHS $2 \cdot 2$, these intakes were not accounted for in the present study.

The CCHS $2 \cdot 2$ also gathered physical measurements of the participants, as well as data on selected health conditions and socio-economic and demographic characteristics of respondents. Key demographic variables were examined to observe the demographic of the average Canadian pulse consumer. Sex was examined as well as age, and a new variable was created, splitting the respondents into one of four age groups: $19-30$ years, $31-50$ years, 51-70 years and $70+$ years. The cultural background variable placed respondents into categories based on their cultural or racial origin and the education level placed respondents into one of four categories based on their highest level of education attained. Income also was examined, splitting the respondents into four groups based on their income adequacy. The classifications were as follows: 'lowest income' for one or two individuals with an income of $\leq \$ 15000$, three or four individuals with an income of $\leq \$ 20000$, or greater than five individuals with an income $\leq \$ 30000$; 'lower middle income' for one or two individuals with an income between $\$ 15000$ and 29999 , three or four individuals with an income between $\$ 20000$ and 39999 or greater than five individuals with an income between $\$ 30000$ and 59999; 'upper middle income' for one or two individuals with an income between $\$ 30000$ and 59999, three or four individuals with an income between $\$ 40000$ and 79999 , or greater than five individuals with an income between $\$ 60000$ and 79999; and 'highest income' for one or two individuals with an income $\geq \$ 60000$, or three or four individuals with an income $\geq \$ 80000^{(23)}$.

To identify all foods and food sources that contained pulses, the following files were used: the food and ingredient details file, the food description file and the food recipe level file. Soyabeans and fresh beans were excluded from the analysis according to the FAO definition of pulses used in the present paper. Food sources included varieties of dry beans (Phaseolus vulgaris) such as the pinto bean, navy bean, kidney bean and black bean, as well as the mung bean (Vigna radiata) and peas which included yellow peas (Lathyrus aphaca), split green peas (Pisum sativum), chickpeas (Cicer arietinum), black-eyed peas (Vigna unguiculata) and lentils (Lens culinaris) $^{(27)}$. Pulse consumers were defined as respondents who consumed pulses or a pulse-containing product for the $1 \mathrm{~d}$ dietary intakes. Consumers were further divided into quartiles based on their level of consumption. Data for macro- and micronutrients were expressed as absolute 
values, percentage energy or quantity per $4184 \mathrm{~kJ}$ (1000 kcal). The percentage of consumers and non-consumers with nutrient intakes below the Institute of Medicine's Dietary Reference Intakes for nutrients that had an estimated average requirement (EAR) were calculated ${ }^{(28)}$. To identify the amount of pulse product per $100 \mathrm{~g}$ of food the Canadian Nutrient File, version 2007b, recipe database and the US Department of Agriculture (USDA) Food and Nutrient Databases for Dietary Studies, version 1.0, were used to find ingredient proportions and process intake data for nutrient components. Food group intake data were obtained from the Canada Food Guide file $^{(23)}$. Further details on both the Canadian Nutrient File database and the USDA Food and Nutrient Database are available on their respective websites ${ }^{(26,29)}$.

General linear models were used to analyse macronutrient and micronutrient intakes and to compare nutrient intakes and other variables between non-consumers and consumers as well as between non-consumers and consumers at each of the four levels of consumption. In addition, similar analyses were conducted for each of the food groups using the data from the CCHS's Canada Food Guide file. Logistic regression was used to determine whether any demographic variables (sex, age, culture, province of residence, income adequacy and education level) increased the likelihood of being classified as a pulse consumer and OR were calculated. Cross-tabulations and $\chi^{2}$ tests were used to compare the proportions of consumers and non-consumers who had intakes of nutrients below their respective EAR values. The significance level was set at $P<0.05$ for differences and $0.05<P<0 \cdot 10$ for trends. All analyses were performed using PASW SPSS Statistics (version 18; IBM) and SUDAAN Statistical Analysis Software Package (version 10.0.1; RTI International).

Because the CCHS 2.2 is a multi-stage survey design, it requires a more complex formula to calculate variance estimates. The approximation method used by the CCHS $2 \cdot 2$ is called 'bootstrapping'. This method is used to estimate standard errors, coefficients of variation and CI. Bootstrapping is an approach used to estimate distribution from a sample's statistics. It also can be defined as 'sampling within a sample' and involves the selection of random samples known as replicates, and the calculation of the variation in the estimates from replicate to replicate ${ }^{(30-32)}$. The bootstrapping method was used in all the data analyses for the present study via SUDAAN software.

\section{Results}

\section{Food sources of pulse products}

The main sources of pulses in the adult Canadian diet were mung beans, Mexican or Hispanic mixed dishes, kidney beans, baked beans, bean soups and chilli. These seven dishes made up two-thirds of the twenty-two dishes containing pulses in the Canadian diet mentioned in the $1 \mathrm{~d}$ recall data (Table 1 ).

\section{Frequency of consumption and demographics}

On any given day, $13.1 \%$ of Canadian adults in 2004 consumed dry beans, peas or lentils (Table 2). Within pulse
Table 1. Food sources of pulse products in the adult Canadian diet*

\begin{tabular}{lc}
\hline Food source & Percentage of source reported \\
\hline Mung beans & $18 \cdot 2$ \\
Mexican or other Hispanic dishes & $14 \cdot 0$ \\
Kidney beans & $13 \cdot 3$ \\
Baked beans & $11 \cdot 4$ \\
Bean soups & $10 \cdot 7$ \\
Chilli & $7 \cdot 4$ \\
Lentils & $5 \cdot 3$ \\
Chickpeas & $3 \cdot 2$ \\
Split peas & $3 \cdot 0$ \\
White beans & $2 \cdot 9$ \\
Hummus & $1 \cdot 8$ \\
Pinto beans & $1 \cdot 3$ \\
Refried beans & $1 \cdot 3$ \\
Black beans & $1 \cdot 2$ \\
Other bean sourcesł & $<1$
\end{tabular}

*According to $1 \mathrm{~d} 24 \mathrm{~h}$ dietary recall of the Canadian Community Health Survey, Cycle $2 \cdot 2$ (2004) of Canadian adults aged $\geq 19$ years. $\dagger$ Excluding chilli.

† Includes rice with beans, navy beans, falafel, unspecified beans, bean dip, adzuki beans, winged beans, and noodles with beans, which each represent less than $1 \%$ of food sources reported.

consumers, average pulse intake was highest in New Brunswick and lowest in Quebec, with the provinces of Ontario and British Columbia having the highest proportions of pulse consumers as residents. The highest proportion of consumers fell into the 51-70 years age bracket (Table 2). Pulse consumption in grams also differed between age groups, but not when expressed relative to energy intake (data not shown).

Participants who identified themselves as Asian Canadian compared with Caucasian were 3.6 times more likely to be pulse consumers. As well, participants who identified themselves as being Arabic, Latin and African Canadian or of multiple cultural origins were 1.6 times more likely to be pulse consumers than Caucasians. Sex, income and education level were not significant determinants of pulse intake (Table 2).

\section{Effects on nutrient intake}

When examining differences in dietary intakes across quartiles of consumption and comparing them with non-consumers (Table 3), consumers in the third $(99 \mathrm{~g} / \mathrm{d})$ and fourth quartile $(294 \mathrm{~g} / \mathrm{d})$ of pulse consumption consumed $937 \mathrm{~kJ}(224 \mathrm{kcal})$ or $11 \%$ and $1360 \mathrm{~kJ}$ (325 kcal) or $16 \%$ more energy per $\mathrm{d}$, respectively, than the average non-consumer. Pulse consumers in the third and fourth quartiles consumed 13 and $24 \%$ more carbohydrate and 12 and 19\% more protein, respectively, than non-consumers, while fat intake was only higher in the third quartile of pulse consumers (by $12 \%$ more than non-consumers). The higher fat intake in pulse consumers in the third quartile was consistent with the higher MUFA, PUFA, linolenic and linoleic fatty acid content in the diets of these individuals. In addition, $\alpha$-linolenic acid intake was $44 \%$ higher in the fourth quartile of pulse consumers compared with non-consumers. Fibre intake was increased the most by pulse consumption, being 34 and $85 \%$ higher, respectively, in the third and fourth quartile of pulse intake. Cholesterol intake was 18\% higher in the second quartile of pulse intake, but not at the other levels of pulse intake. 
Table 2. Demographic characteristics of pulse consumers and non-consumers based on $1 \mathrm{~d}$ intakes from the Canadian Community Health Survey, Cycle 2.2 (2004)

(Odds ratios and $95 \%$ confidence intervals; mean values and standard errors)

\begin{tabular}{|c|c|c|c|c|c|}
\hline \multirow[b]{2}{*}{ Characteristic } & \multirow{2}{*}{$\begin{array}{c}\text { Proportion of } \\
\text { consumers (\%) }(n 20156)\end{array}$} & \multirow[b]{2}{*}{ OR } & \multirow[b]{2}{*}{$95 \% \mathrm{Cl}$} & \multicolumn{2}{|c|}{$\begin{array}{l}\text { Amount of pulses } \\
\text { consumed }(\mathrm{g} / \mathrm{d})\end{array}$} \\
\hline & & & & Mean & SE \\
\hline \multicolumn{6}{|l|}{ Sex } \\
\hline Male & $13 \cdot 1$ & 1 & Reference & $120 \cdot 1$ & $6 \cdot 1$ \\
\hline Female & $13 \cdot 2$ & 1.01 & $0.8,1.29$ & $105 \cdot 1$ & $10 \cdot 1$ \\
\hline \multicolumn{6}{|l|}{ Age (years) } \\
\hline $19-30$ & 11.5 & 1 & Reference & 131.9 & 29.5 \\
\hline $31-50$ & $13 \cdot 2$ & 1.17 & $0.84,1.63$ & 114.9 & $12 \cdot 3$ \\
\hline $51-70$ & $14 \cdot 7$ & 1.32 & $1.03,1.69$ & $105 \cdot 3^{\star}$ & 13.7 \\
\hline $70+$ & $12 \cdot 2$ & 1.06 & $0.77,1.47$ & $86 \cdot 2$ & $10 \cdot 0$ \\
\hline \multicolumn{6}{|l|}{ Provincial location } \\
\hline Nova Scotia & $8 \cdot 2$ & 1 & Reference & $124 \cdot 3 \dagger$ & $15 \cdot 0$ \\
\hline Newfoundland and Labrador & $12 \cdot 6$ & 1.61 & $0.96,2.71$ & $125 \cdot 1$ & $20 \cdot 8$ \\
\hline Prince Edward Island & $11 \cdot 3$ & 1.42 & $0.78,2.59$ & $109 \cdot 7$ & $21 \cdot 8$ \\
\hline New Brunswick & $10 \cdot 9$ & 1.37 & $0.74,2.53$ & $145 \cdot 8 \dagger$ & 34.9 \\
\hline Quebec & $10 \cdot 4$ & $1 \cdot 30$ & $0.78,2 \cdot 16$ & $82 \cdot 6$ & $7 \cdot 8$ \\
\hline Ontario & 14.5 & 1.89 & $1 \cdot 18,3.05$ & $128 \cdot 2 \nmid \dagger$ & 23.4 \\
\hline Manitoba & $12 \cdot 9$ & 1.66 & $0.89,3.1$ & $105 \cdot 7$ & $12 \cdot 4$ \\
\hline Saskatchewan & $11 \cdot 3$ & 1.43 & $0.78,2.63$ & $98 \cdot 8$ & $21 \cdot 4$ \\
\hline Alberta & $11 \cdot 3$ & 1.43 & $0.75,2.74$ & $96 \cdot 0$ & $25 \cdot 9$ \\
\hline British Columbia & $17 \cdot 6$ & $2 \cdot 39$ & $1 \cdot 35,4.25$ & $111.4 \dagger \dagger$ & $8 \cdot 7$ \\
\hline All of Canada & $13 \cdot 1$ & & & $112 \cdot 4$ & $7 \cdot 2$ \\
\hline \multicolumn{6}{|l|}{ Culture } \\
\hline Caucasian & $10 \cdot 8$ & 1 & Reference & $104 \cdot 8$ & $5 \cdot 1$ \\
\hline African Canadian, Arab, Latin, multiple origins & $16 \cdot 5$ & 1.64 & $1.05,2.56$ & 129.5 & 34.4 \\
\hline Asian Canadian & $30 \cdot 4$ & 3.62 & $2 \cdot 04,6 \cdot 42$ & $135 \cdot 1$ & 37.4 \\
\hline Aboriginal & $11 \cdot 1$ & 1.04 & $0.45,2 \cdot 38$ & $90 \cdot 9$ & 33.1 \\
\hline Other & $23 \cdot 2$ & $2 \cdot 5$ & $0.94,6.71$ & $76 \cdot 5$ & 44.9 \\
\hline \multicolumn{6}{|l|}{ Income } \\
\hline Lowest & $13 \cdot 7$ & 1 & Reference & $113 \cdot 3$ & $19 \cdot 3$ \\
\hline Lower middle & $14 \cdot 1$ & 1.04 & $0.52,2.05$ & $105 \cdot 6$ & 8.9 \\
\hline Upper middle & $12 \cdot 6$ & 0.91 & $0.47,1.78$ & $139 \cdot 8$ & $30 \cdot 7$ \\
\hline Highest & 13.5 & 0.98 & $0.47,2.03$ & 94.2 & $18 \cdot 1$ \\
\hline \multicolumn{6}{|l|}{ Education } \\
\hline$<$ Secondary school & $11 \cdot 0$ & 1 & Reference & $104 \cdot 3$ & $6 \cdot 0$ \\
\hline Secondary school & $12 \cdot 6$ & $1 \cdot 16$ & $0.71,1.9$ & $120 \cdot 2$ & $14 \cdot 2$ \\
\hline Post-secondary school & $10 \cdot 3$ & 0.93 & $0.62,1.39$ & $92 \cdot 0$ & 13.3 \\
\hline Post-secondary degree or diploma & 14.5 & 1.37 & $0.93,1.56$ & $115 \cdot 0$ & $10 \cdot 6$ \\
\hline
\end{tabular}

*Mean value was marginally significantly different from that of the $70+$ years age group $(0.05<P<0 \cdot 10)$.

Mean value was (marginally) significantly different from that of consumers of the province of Quebec: $† 0.05<P<0.10, \dagger \dagger P<0.05$.

However, when expressed relative to energy intake, cholesterol levels were lowest in the highest quartile of pulse consumption. A number of micronutrients were higher in the highest pulse consumers compared with non-consumers (Table 3). These included folate (45\%), P (20\%), Mg (35\%), Fe $(35 \%)$ and $\mathrm{Zn}(28 \%)$. As a result, the proportion of those consuming these nutrients below the EAR was lower in consumers compared with non-consumers, indicating a reduced level of deficiency in consumers (Table 4). $\mathrm{K}$ intake also was higher among pulse consumers, and, although it lacks an EAR, analysis using adequate intake levels showed no significant difference between consumers and non-consumers (data not shown). In contrast, the levels of two vitamins were lower in the fourth quartile of pulse consumers compared with non-consumers: vitamin D (20\%), and vitamin $\mathrm{B}_{12}$ (14\%). However, the proportion of those that were below the EAR for these two nutrients was similar for both consumers and non-consumers. Finally, Na intake was $31 \%$ higher in pulse consumers $v$. non-consumers.
Fruit and vegetable consumption was higher in the higher quartiles of pulse consumption, when compared with nonconsumers, resulting in approximately one more serving from this food group. There was no difference in the amount of total grain products or dairy products between pulse consumers and non-consumers. In the two highest quartiles of pulse consumers, intakes from the meat and alternatives food groups were $1-3.5$ servings higher when compared with non-consumers, consistent with the inclusion of pulses in this food group by Health Canada in this database (Fig. 1).

\section{Discussion}

The proportion of pulse consumers in Canadian adults appears to be similar to that of the US population, despite important differences in cultural makeup. We previously reported that $7.9 \%$ of the US populations are pulse consumers, but the NHANES analysis did not include mung beans or other sprouted forms of beans (such as navy bean sprouts) 
Table 3. Pulse amount and macronutrient, micronutrient and energy intakes per $d$ for non-consumers and by quartiles $(Q)$ of pulse consumers based on $1 \mathrm{~d}$ intakes from the Canadian Community Health Survey Cycle $2 \cdot 2$ (2004)

(Mean values with their standard errors)

\begin{tabular}{|c|c|c|c|c|c|c|c|c|c|c|}
\hline \multirow{3}{*}{ Intake category. ... } & \multirow{2}{*}{\multicolumn{2}{|c|}{$\begin{array}{l}\text { Non-consumers } \\
(n 17750)\end{array}$}} & \multicolumn{8}{|c|}{ Consumers ( $n$ 2406) } \\
\hline & & & \multicolumn{2}{|c|}{ Q1 } & \multicolumn{2}{|c|}{ Q2 } & \multicolumn{2}{|c|}{ Q3 } & \multicolumn{2}{|c|}{ Q4 } \\
\hline & Mean & SE & Mean & SE & Mean & SE & Mean & SE & Mean & SE \\
\hline Pulse intake range $(\mathrm{g})$ & \multicolumn{2}{|c|}{0} & \multicolumn{2}{|c|}{$0-28.89$} & \multicolumn{2}{|c|}{$28 \cdot 90-66.29$} & \multicolumn{2}{|c|}{$66 \cdot 30-137 \cdot 19$} & \multicolumn{2}{|c|}{$>137.2$} \\
\hline Pulse intake $(\mathrm{g})$ & \multirow{2}{*}{\multicolumn{2}{|c|}{0}} & 12.9 & 0.7 & $47 \cdot 2$ & $1 \cdot 1$ & $99 \cdot 1$ & $2 \cdot 4$ & 293.9 & 39.8 \\
\hline Pulses (g/4184kJ)‡ & & & $6 \cdot 1$ & $1 \cdot 2$ & $22 \cdot 8$ & 1.2 & 38.5 & $5 \cdot 3$ & $75 \cdot 6$ & 3.4 \\
\hline Food amount (g) & 3219 & 119 & 3540 & 441 & 3280 & 174 & 3315 & 139 & $3619 \dagger$ & 337 \\
\hline Energy & & & & & & & & & & \\
\hline $\mathrm{kJ}$ & 8640 & 561 & 9535 & 1331 & 8895 & 347 & $9619^{*}$ & 556 & $10000^{\star \star}$ & 494 \\
\hline kcal & 2065 & 134 & 2279 & 318 & 2126 & 83 & $2299^{*}$ & 133 & $2390^{\star *}$ & 118 \\
\hline Macronutrients & & & & & & & & & & \\
\hline Carbohydrate (g) & 253 & 16 & 280 & 38 & 256 & 12 & $286^{\star \star \star}$ & 16 & $314^{\star \star *}$ & 25 \\
\hline Protein $(\mathrm{g})$ & 84.5 & $5 \cdot 3$ & 94.3 & $12 \cdot 6$ & 91.9 & 4.5 & $94.9^{*}$ & 7.9 & $100 \cdot 7^{\star \star \star}$ & $6 \cdot 7$ \\
\hline Total fat (g) & $75 \cdot 6$ & $5 \cdot 1$ & $81 \cdot 2$ & $12 \cdot 2$ & $77 \cdot 8$ & 3.6 & $84.5^{\star}$ & $6 \cdot 4$ & 78.9 & $6 \cdot 1$ \\
\hline SFA $(g)$ & 24.8 & $2 \cdot 0$ & $24 \cdot 8$ & 3.1 & $22 \cdot 8$ & 1.6 & $26 \cdot 2$ & $2 \cdot 3$ & $23 \cdot 6$ & $2 \cdot 2$ \\
\hline MUFA (g) & $30 \cdot 3$ & $2 \cdot 1$ & 32.9 & $5 \cdot 3$ & $32 \cdot 1$ & 1.5 & $34 \cdot 6^{*}$ & $2 \cdot 6$ & $32 \cdot 9$ & 2.9 \\
\hline PUFA (g) & $13 \cdot 3$ & 0.6 & $15 \cdot 9$ & 3.0 & $15 \cdot 7$ & 0.9 & $16 \cdot 2^{*}$ & 1.7 & 14.9 & 0.9 \\
\hline Linoleic fatty acid (g) & $10 \cdot 6$ & 0.4 & $12 \cdot 9$ & $2 \cdot 9$ & $12 \cdot 4$ & 0.7 & $12 \cdot 8^{*}$ & 1.4 & $11 \cdot 7$ & 0.8 \\
\hline Linolenic fatty acid (g) & 1.9 & 0.09 & $2 \cdot 3$ & 0.32 & $2 \cdot 4$ & 0.18 & $2 \cdot 5^{\star}$ & 0.34 & $2 \cdot 6^{\star \star}$ & 0.28 \\
\hline Carbohydrate (\% kJ) & $49 \cdot 1$ & 0.3 & 49.4 & 0.9 & $48 \cdot 7$ & 1.4 & 49.7 & 0.9 & $52 \cdot 4$ & 1.9 \\
\hline Protein $(\% \mathrm{~kJ})$ & 16.5 & 0.1 & $16 \cdot 7$ & 0.5 & $17 \cdot 2 \dagger$ & 0.4 & $16 \cdot 5$ & 0.6 & $16 \cdot 9$ & 0.6 \\
\hline Fat $(\% \mathrm{~kJ})$ & 31.5 & 0.2 & $30 \cdot 6$ & 0.7 & 31.5 & $1 \cdot 2$ & 31.4 & 0.9 & $28 \cdot 0$ & $2 \cdot 5$ \\
\hline SFA (\% kJ) & $10 \cdot 3$ & 0.2 & $9.4 \dagger$ & 0.4 & $9.1^{*}$ & 0.4 & $9 \cdot 7^{*}$ & 0.4 & $8.3 \dagger$ & 1.0 \\
\hline MUFA (\% kJ) & 12.5 & 0.1 & 12.4 & 0.4 & 12.9 & 0.5 & $12 \cdot 9$ & 0.4 & 11.6 & 1.1 \\
\hline PUFA (\% kJ) & $5 \cdot 5$ & 0.2 & $5 \cdot 8$ & 0.2 & $6 \cdot 5^{\star \star \star}$ & 0.3 & $6 \cdot 0$ & 0.3 & $5 \cdot 3$ & 0.3 \\
\hline Linoleic fatty acid (\% kJ) & 4.4 & 0.1 & 4.6 & 0.3 & $5 \cdot 1^{\star *}$ & 0.2 & 4.7 & 0.3 & 4.2 & 0.3 \\
\hline Linolenic fatty acid (\% kJ) & 0.8 & 0.02 & $0.9^{*}$ & 0.05 & $1.0^{\star \star}$ & 0.08 & 1.0 & 0.11 & 0.9 & 0.09 \\
\hline Cholesterol (mg) & 279 & 19 & $316 \dagger$ & 33 & $330^{*}$ & 32 & 303 & 34 & 239 & 36 \\
\hline Cholesterol (mg/4184 kJ) & 139 & 2 & 140 & 11 & 150 & 9 & 131 & 10 & $103^{*}$ & 13 \\
\hline Fibre (g) & $16 \cdot 6$ & 0.4 & $18 \cdot 1$ & 1.67 & $18 \cdot 7$ & 1.13 & $22 \cdot 2^{\star \star *}$ & 1.6 & $30 \cdot 7^{\star \star \star}$ & $2 \cdot 2$ \\
\hline Fibre $(\mathrm{g} / 4184 \mathrm{~kJ})$ & 8.5 & 0.4 & 8.5 & 0.6 & 9.4 & 0.6 & $10.5^{\star \star *}$ & 0.4 & $13 \cdot 8^{\star \star \star}$ & 0.7 \\
\hline Micronutrients & & & & & & & & & & \\
\hline Vitamin D $(\mu \mathrm{g})$ & $5 \cdot 7$ & 0.1 & $6 \cdot 0$ & 0.8 & $6 \cdot 0$ & 1.0 & $6 \cdot 3$ & 1.5 & $4 \cdot 6^{\star}$ & 0.4 \\
\hline Vitamin D ( $\mu \mathrm{g} / 4184 \mathrm{~kJ})$ & $2 \cdot 8$ & 0.2 & $2 \cdot 7$ & 0.3 & 2.9 & 0.4 & $2 \cdot 7$ & 0.9 & $2 \cdot 0^{\star \star \star}$ & 0.2 \\
\hline Thiamin (mg) & 1.7 & 0.06 & 1.8 & 0.26 & $1 \cdot 7$ & 0.08 & $1.9^{\star}$ & 0.15 & 1.90 & 0.23 \\
\hline Thiamin (mg/4184 kJ) & 0.9 & 0.04 & 0.8 & 0.03 & 0.8 & 0.03 & 0.8 & 0.03 & 0.8 & 0.10 \\
\hline Riboflavin (mg) & 1.9 & 0.10 & $2 \cdot 1$ & 0.25 & 1.9 & 0.08 & $2 \cdot 1 \dagger$ & 0.14 & $2 \cdot 0$ & 0.09 \\
\hline Riboflavin (mg/4184 kJ) & 0.96 & 0.02 & 0.94 & 0.03 & 0.95 & 0.03 & 0.93 & 0.03 & $0.87^{* *}$ & 0.02 \\
\hline Niacin (mg) & $39 \cdot 6$ & 2.5 & 43.3 & $5 \cdot 8$ & $42 \cdot 7$ & $2 \cdot 0$ & $43 \cdot 1^{*}$ & $2 \cdot 7$ & 43.9 & $2 \cdot 3$ \\
\hline Niacin (mg/4184 kJ) & $19 \cdot 7$ & 0.1 & 19.7 & 0.6 & $20 \cdot 6$ & 0.5 & $19 \cdot 0$ & 0.5 & $19 \cdot 0$ & 0.5 \\
\hline Vitamin $B_{6}(\mathrm{mg})$ & 1.87 & 0.1 & 2.09 & 0.3 & 1.99 & 0.1 & $2.09 \dagger$ & 0.1 & $2 \cdot 12^{\star \star}$ & 0.1 \\
\hline Vitamin $\mathrm{B}_{6}(\mathrm{mg} / 4184 \mathrm{~kJ})$ & 0.94 & 0.02 & 0.97 & 0.03 & 0.99 & 0.05 & 0.95 & 0.06 & 0.91 & 0.03 \\
\hline Vitamin $B_{12}(\mathrm{mg})$ & 4.4 & 0.3 & $5 \cdot 1$ & 0.7 & 4.9 & 0.7 & $4 \cdot 2$ & 1.0 & $3 \cdot 8^{\star}$ & 0.3 \\
\hline Vitamin $B_{12}(\mathrm{mg} / 4184 \mathrm{~kJ})$ & $2 \cdot 2$ & 0.04 & $2 \cdot 2$ & 0.32 & $2 \cdot 4$ & 0.63 & 1.9 & 0.60 & $1 \cdot 7^{\star \star}$ & 0.16 \\
\hline Folate§ $(\mu \mathrm{g})$ & 453 & $34 \cdot 1$ & 470 & 66.6 & 450 & $16 \cdot 1$ & $541 t$ & $80 \cdot 3$ & $656^{* \star *}$ & 59.0 \\
\hline Folate§ ( $\mu \mathrm{g} / 4184 \mathrm{~kJ})$ & 229 & $3 \cdot 7$ & 216 & $6 \cdot 3$ & 218 & $10 \cdot 0$ & 241 & $16 \cdot 3$ & $289^{* \star *}$ & $17 \cdot 0$ \\
\hline Folic acid\| $(\mu \mathrm{g})$ & $119 \cdot 8$ & 18.95 & $157 \cdot 3$ & 43.4 & $130 \cdot 2$ & $28 \cdot 0$ & $139 \cdot 0$ & $32 \cdot 1$ & $133 \cdot 2$ & 20.7 \\
\hline Folic acid $\|(\mu \mathrm{g} / 4184 \mathrm{~kJ})$ & 58.5 & 5.8 & 68.0 & $10 \cdot 12 \dagger$ & $59 \cdot 7$ & $9 \cdot 8$ & 58.9 & $7 \cdot 8$ & $56 \cdot 3$ & 8.50 \\
\hline $\mathrm{Ca}(\mathrm{mg})$ & 865 & $62 \cdot 3$ & 885 & 93.5 & 749 & 52.5 & 937 & $69 \cdot 8$ & $953.8 \dagger$ & 92.9 \\
\hline $\mathrm{Ca}(\mathrm{mg} / 4184 \mathrm{~kJ})$ & 432 & 4.1 & 405 & 18.9 & $369.5^{\star \star \star}$ & $16 \cdot 0$ & $416 \cdot 4$ & 15.8 & 402 & $26 \cdot 1$ \\
\hline$P(\mathrm{mg})$ & 1330 & $63 \cdot 1$ & 1454 & $186 \cdot 9$ & 1352 & $61 \cdot 8$ & $1497^{\star *}$ & 84.4 & $1597^{\star \star \star}$ & 107.9 \\
\hline $\mathrm{P}(\mathrm{mg} / 4184 \mathrm{~kJ})$ & 660 & 11.4 & 650 & 17.8 & 657 & 18.9 & 671 & $19 \cdot 1$ & 687 & 25.0 \\
\hline $\mathrm{Mg}(\mathrm{mg})$ & 323 & $14 \cdot 2$ & 359 & $45 \cdot 6$ & 339 & $14 \cdot 8$ & $375^{\star \star \star}$ & $17 \cdot 1$ & $438^{\star \star \star}$ & 39.5 \\
\hline $\mathrm{Mg}(\mathrm{mg} / 4184 \mathrm{~kJ})$ & 166 & 4.5 & 166 & 3.7 & 171 & 5.5 & $175 \dagger$ & $4 \cdot 8$ & $192 \dagger$ & 9.7 \\
\hline $\mathrm{Fe}(\mathrm{mg})$ & $13 \cdot 9$ & 0.7 & $14 \cdot 8$ & 1.6 & $14 \cdot 1$ & 0.5 & $16 \cdot 04^{\star * *}$ & 0.7 & $18 \cdot 7^{\star \star \star}$ & 0.8 \\
\hline $\mathrm{Fe}(\mathrm{mg} / 4184 \mathrm{~kJ})$ & $7 \cdot 0$ & 0.1 & $6 \cdot 8$ & 0.2 & $7 \cdot 0$ & 0.2 & $7 \cdot 2$ & 0.4 & $8 \cdot 3^{\star \star \star}$ & 0.3 \\
\hline $\mathrm{Zn}(\mathrm{mg})$ & 11.2 & 0.6 & 12.5 & 2.0 & 11.8 & 0.8 & $12 \cdot 7^{\star \star}$ & 0.7 & $14 \cdot 3^{\star \star \star}$ & 0.6 \\
\hline $\mathrm{Zn}(\mathrm{mg} / 4184 \mathrm{~kJ})$ & 5.5 & 0.1 & $5 \cdot 6$ & 0.3 & $5 \cdot 7$ & 0.24 & $5 \cdot 7$ & 0.2 & $6 \cdot 2^{\star \star \star}$ & 0.2 \\
\hline $\mathrm{Na}(\mathrm{mg})$ & 3050 & $169 \cdot 6$ & 3320 & $412 \cdot 0$ & $3369 \dagger$ & $141 \cdot 2$ & $3581 \dagger$ & $202 \cdot 9$ & $3988^{*}$ & $250 \cdot 4$ \\
\hline $\mathrm{Na}(\mathrm{mg} / 4184 \mathrm{~kJ})$ & 1522 & $32 \cdot 6$ & 1503 & 39.5 & $1613 \dagger$ & $57 \cdot 7$ & 1584 & $122 \cdot 4$ & $1721 \dagger$ & $143 \cdot 2$ \\
\hline $\mathrm{K}(\mathrm{mg})$ & 3074 & 93.5 & 3351 & $438 \cdot 4$ & 3192 & $160 \cdot 0$ & 3476 & 133.4 & 3927 & 152.5 \\
\hline $\mathrm{K}(\mathrm{mg} / 4184 \mathrm{~kJ})$ & 1584 & $56 \cdot 4$ & 1564 & 40.5 & 1597 & $86 \cdot 8$ & 1608 & $62 \cdot 7$ & $1708^{\star \star}$ & $66 \cdot 2$ \\
\hline
\end{tabular}

Mean value was significantly different from that of the non-consumer group: ${ }^{*} P<0.05,{ }^{\star \star} P<0.01,{ }^{\star \star \star} * 0<0.001$.

† Mean value was marginally significantly different from that of the non-consumer group $(0.05<P<0.10)$.

$\ddagger 4184 \mathrm{~kJ}$ is equivalent to $1000 \mathrm{kcal}$.

$\S$ Folate intake from food in dietary folate equivalency.

॥ Folic acid is the synthetic form of folate, found in fortified foods. 
Table 4. Prevalence of inadequacy for nutrientsł with an estimated average requirement (EAR) in Canadian adults based on $1 \mathrm{~d}$ intakes from the Canadian Community Health Survey, Cycle $2 \cdot 2$ (2004)

\begin{tabular}{|c|c|c|c|c|}
\hline & \multicolumn{4}{|c|}{ Percentage of intakes less than EAR } \\
\hline & \multicolumn{2}{|c|}{ Male } & \multicolumn{2}{|c|}{ Female§ } \\
\hline & Non-consumers & Consumers & Non-consumers & Consumers \\
\hline \multicolumn{5}{|l|}{$\mathrm{Ca}$} \\
\hline $19-30$ years & $42 \cdot 7$ & 42.9 & 55.0 & $52 \cdot 0$ \\
\hline $31-50$ years & 50.4 & 41.9 & $56 \cdot 8$ & $62 \cdot 1$ \\
\hline $51-70$ years & $57 \cdot 3$ & $56 \cdot 2$ & 78.4 & $75 \cdot 8$ \\
\hline$>70$ years & $76 \cdot 9$ & $73 \cdot 8$ & $82 \cdot 1$ & 83.1 \\
\hline \multicolumn{5}{|l|}{ Vitamin D } \\
\hline $19-30$ years & $85 \cdot 3$ & 84.7 & $90 \cdot 0$ & 89.5 \\
\hline $31-50$ years & $86 \cdot 9$ & $87 \cdot 8$ & 90.5 & $92 \cdot 4$ \\
\hline $51-70$ years & 85.6 & 75.6 & 91.8 & 93.5 \\
\hline$>70$ years & $85 \cdot 3$ & 88.9 & $90 \cdot 7$ & $92 \cdot 6$ \\
\hline \multicolumn{5}{|l|}{ Thiamin } \\
\hline $19-30$ years & $15 \cdot 3$ & $7 \cdot 2$ & $26 \cdot 2 \dagger$ & $16 \cdot 3$ \\
\hline $31-50$ years & $15 \cdot 7$ & $13 \cdot 8$ & $21.9 \dagger$ & $15 \cdot 3$ \\
\hline $51-70$ years & $16 \cdot 4^{\star *}$ & $9 \cdot 1$ & 24.6 & 23.2 \\
\hline$>70$ years & $20 \cdot 0$ & $17 \cdot 5$ & $27 \cdot 5$ & $20 \cdot 9$ \\
\hline \multicolumn{5}{|l|}{ Riboflavin } \\
\hline $19-30$ years & $11 \cdot 7$ & $12 \cdot 2$ & $18 \cdot 6$ & $10 \cdot 0$ \\
\hline $31-50$ years & $11 \cdot 8$ & 11.4 & $13 \cdot 3$ & 9.7 \\
\hline $51-70$ years & 14.0 & 11.3 & $15 \cdot 2$ & $10 \cdot 3$ \\
\hline$>70$ years & $19 \cdot 6$ & $16 \cdot 7$ & $17 \cdot 9$ & $12 \cdot 6$ \\
\hline \multicolumn{5}{|l|}{ Vitamin $\mathrm{B}_{6}$} \\
\hline $19-30$ years & $15 \cdot 7$ & $6 \cdot 2$ & $34.9^{*}$ & $21 \cdot 2$ \\
\hline $31-50$ years & $16 \cdot 2$ & $16 \cdot 9$ & $30 \cdot 4$ & $18 \cdot 8$ \\
\hline $51-70$ years & 32.4 & $24 \cdot 3$ & $41 \cdot 8$ & 33.9 \\
\hline$>70$ years & $39 \cdot 0$ & $28 \cdot 0$ & 42.9 & $36 \cdot 4$ \\
\hline \multicolumn{5}{|l|}{ Folate\| } \\
\hline $19-30$ years & $22 \cdot 2$ & 9.7 & $44 \cdot 2^{\star \star}$ & $22 \cdot 7$ \\
\hline $31-50$ years & $26 \cdot 8^{\star \star}$ & $19 \cdot 0$ & $40 \cdot 6^{\star \star}$ & 29.0 \\
\hline $51-70$ years & $27 \cdot 0^{\star * *}$ & $20 \cdot 0$ & $42 \cdot 8 \dagger$ & $30 \cdot 1$ \\
\hline$>70$ years & 39.5 & $27 \cdot 3$ & 54.1 & 39.5 \\
\hline \multicolumn{5}{|l|}{ Vitamin $\mathrm{B}_{12}$} \\
\hline $19-30$ years & 24.5 & $27 \cdot 4$ & $41 \cdot 1$ & 41.4 \\
\hline $31-50$ years & $21 \cdot 7$ & $22 \cdot 1$ & $36 \cdot 5$ & 39.2 \\
\hline $51-70$ years & 24.5 & $22 \cdot 6$ & 38.7 & $32 \cdot 0$ \\
\hline$>70$ years & 34.6 & $32 \cdot 5$ & $42 \cdot 8$ & 38.5 \\
\hline \multicolumn{5}{|l|}{$\mathrm{Fe}$} \\
\hline $19-30$ years & $5 \cdot 1$ & $1 \cdot 1$ & $27 \cdot 2^{\star}$ & $15 \cdot 2$ \\
\hline $31-50$ years & $4 \cdot 3^{\star \star}$ & 0.8 & $26 \cdot 1 \dagger$ & $12 \cdot 7$ \\
\hline $51-70$ years & $5 \cdot 0^{\star \star \star *}$ & $2 \cdot 0$ & $7 \cdot 2$ & 3.4 \\
\hline$>70$ years & $8 \cdot 2$ & 3.4 & $7 \cdot 0$ & $4 \cdot 1$ \\
\hline \multicolumn{5}{|l|}{$\mathrm{Mg}$} \\
\hline $19-30$ years & 49.7 & 31.8 & $49 \cdot 1^{*}$ & $31 \cdot 6$ \\
\hline $31-50$ years & $56 \cdot 7$ & $40 \cdot 8$ & $47.3 \dagger$ & 31.9 \\
\hline $51-70$ years & $60 \cdot 6^{\star \star \star}$ & $45 \cdot 1$ & $46.3 \dagger$ & $32 \cdot 1$ \\
\hline$>70$ years & $66 \cdot 7^{\star \star *}$ & $55 \cdot 2$ & $53 \cdot 1$ & $36 \cdot 4$ \\
\hline \multicolumn{5}{|c|}{$\mathrm{P}$} \\
\hline $19-30$ years & $5 \cdot 6$ & 1.2 & $13.7 \dagger$ & $5 \cdot 3$ \\
\hline $31-50$ years & 4.6 & 4.7 & $11 \cdot 1 \dagger$ & $5 \cdot 3$ \\
\hline $51-70$ years & $5 \cdot 8^{\star \star \star}$ & 4.4 & $12 \cdot 1^{\circ}$ & $6 \cdot 9$ \\
\hline$>70$ years & $10 \cdot 0$ & $10 \cdot 2$ & $14 \cdot 9$ & $9 \cdot 3$ \\
\hline \multicolumn{5}{|c|}{$\mathrm{Zn}$} \\
\hline $19-30$ years & $33 \cdot 3$ & $17 \cdot 8$ & $36 \cdot 6^{*}$ & $21 \cdot 7$ \\
\hline $31-50$ years & $35 \cdot 1^{*}$ & $20 \cdot 4$ & $35 \cdot 1^{\star}$ & $18 \cdot 7$ \\
\hline $51-70$ years & $42 \cdot 7^{\star \star \star}$ & 28.2 & $34 \cdot 8$ & 23.0 \\
\hline$>70$ years & $53 \cdot 5^{\star}$ & 39.0 & $43 \cdot 3$ & $28 \cdot 4$ \\
\hline
\end{tabular}

Mean value was significantly different from that of non-consumers in the same life-stage group: ${ }^{*} P<0.05,{ }^{* *} P<0.01,{ }^{* \star *} P<0.001$. † Mean value was marginally significantly different from that of non-consumers in the same life-stage group $(0.05<P<0.10)$. $\ddagger$ Not including $\mathrm{K}$ (which lacks an EAR) and niacin (analysis showed no significant difference between groups).

$\S$ Excluding pregnant or lactating women.

|| Folate intake from food in dietary folate equivalency. 


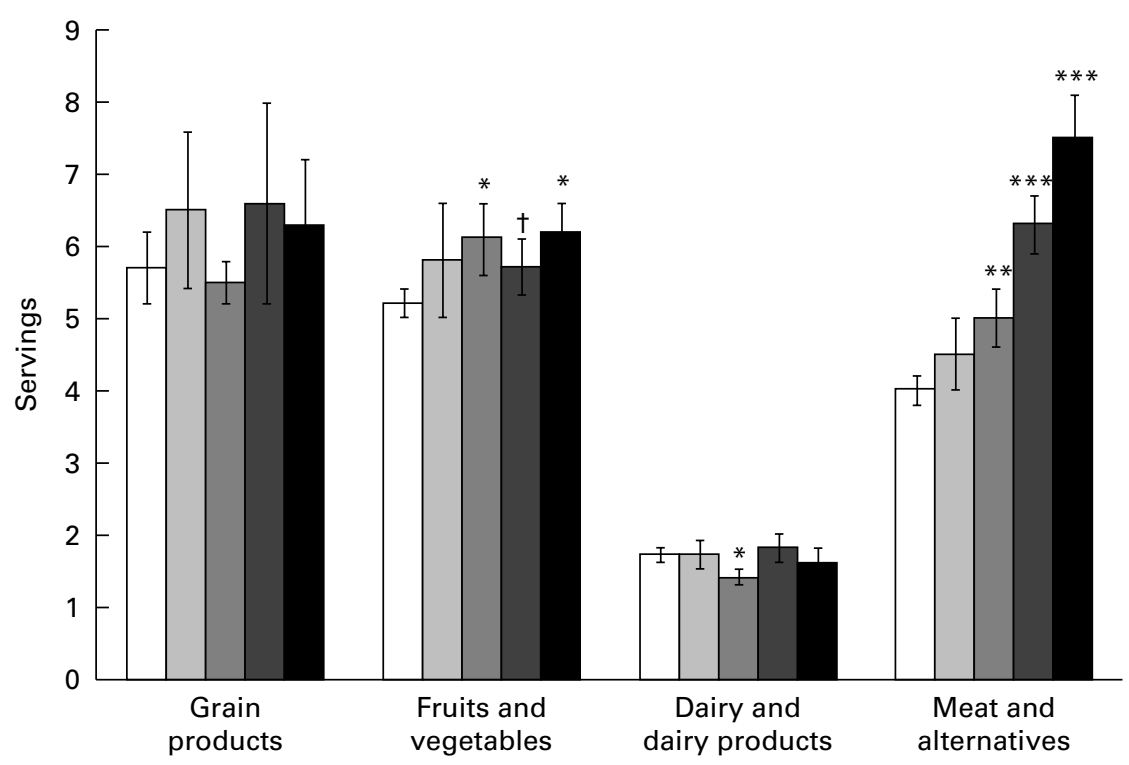

Food groups

Fig. 1. Food group intakes among non-consumers $(\square)$ and by quartiles ( $\square$, quartile $1 ; \square$, quartile 2; $\square$, quartile 3; $\mathbf{\square}$, quartile 4) of pulse consumers based on 1 d intakes from the Canadian Community Health Survey Cycle 2.2 (2004). Food groups and serving sizes are from the Canada Food Guide ${ }^{(23)}$. Pulses are included in the meat and alternatives group. Values are means, with standard errors represented by vertical bars. Mean value was significantly different from that of the non-consumer group: ${ }^{\star} P<0.05,{ }^{* *} P<0.01,{ }^{\star * *} P<0.001$. †Mean value was marginally significantly different from that of the non-consumer group $(0.05<P<0.10)$

as a source of pulse consumption ${ }^{(22)}$. When these were removed from our pulse database, the proportion of the Canadian adult population classified as pulse consumers dropped from $13 \cdot 1 \%$ to $10 \cdot 7 \%$. In the Continuing Survey of Food Intakes by Individuals 1994-1996, 14\% of US residents consumed at least one pulse-containing food over a $2 \mathrm{~d}$ period $^{(33)}$. Similarly in our analysis, when the smaller subset of 10786 respondents with both day 1 and day 2 recalls were combined, $14.7 \%$ were pulse consumers. When the smaller subset of respondents who completed the day 2 recall was examined alone, the rate of consumption was $12.6 \%$. Other differences between these studies include larger sample size for the CCHS $2 \cdot 2$ data and the inclusion of foods such as lima beans (which we excluded in our analyses), and exclusion of lentils, split peas and yellow peas, which represented approximately $8 \%$ of the food sources reported in the CCHS $2 \cdot 2$ survey.

In terms of the most frequently consumed pulse-containing foods, in the NHANES study pinto beans and refried beans were predominantly consumed, which mimicked results found in the Continuing Survey of Food Intakes by Individuals study $^{(33)}$. In Canada, however, pinto and refried beans represented only $1 \%$ of the pulse foods reported, while mung beans were the most popular pulse in the CCHS $2 \cdot 2$ dataset. This probably can be ascribed to the differences in the cultural mosaic of the USA and Canada. The US Hispanic population represents $16 \%$ of the US population ${ }^{(34)}$, while the same group represents approximately $1 \%$ of the Canadian population $^{(35)}$. Conversely, the Asian population in Canada is approximately $10 \%$, making up approximately $66 \%$ of Canada's visible minority population ${ }^{(35,36)}$, while Asian
Americans represent less than $5 \%$ of the US population ${ }^{(37)}$ The provinces with the highest proportion of Asians, Ontario and British Columbia, were also the two provinces found to contain the highest proportion of pulse consumers ${ }^{(35)}$, fitting in with the finding that Asian Canadians were found to be almost four times more likely to be pulse consumers. In contrast to the US data, neither education level nor income level influenced pulse consumption in Canadians, perhaps reflecting the differing cultural backgrounds of Canadian consumers, or the higher proportion of US citizens (27\%) having less than a high school education compared with Canadians $(16 \%)^{(22,33)}$

Overall, consumption of pulses is associated with improved nutrient intakes, especially in the highest quartile of intake. Similar effects on dietary quality were observed in our previous analysis of the NHANES population ${ }^{(22)}$. The nutrient intake which improved the most with pulse consumption was fibre. Consumers in the highest quartiles of pulse consumption in both Canada and the USA consume almost twice as much fibre as non-consumers. Although this level of intake still does not meet the dietary recommendation for this nutrient, it should be noted that the Canadian Nutrient File lacks data on functional fibre (i.e. isolated, extracted or synthetic fibre) so it is possible that the fibre intakes may be underestimated in this analysis.

The higher intakes of carbohydrate, protein and fibre are probably due to these macronutrients being present in large amounts in pulses. This also contributes to the improvement of diet quality observed in pulse consumers, as $32 \%$ of males and $21 \%$ of females, aged 19 years and older, have carbohydrate intakes below the acceptable macronutrient distribution range ${ }^{(38-40)}$. Several micronutrients (folate, $\mathrm{Mg}, \mathrm{Fe}$, 
$\mathrm{K}$ and $\mathrm{Zn}$ ) that were consumed by pulse eaters in larger amounts also can be explained by these nutrients being found in higher levels in pulses ${ }^{(6,41)}$. Indeed, there were fewer pulse consumers that had inadequate intakes (below the EAR) of $\mathrm{Mg}, \mathrm{Zn}$, folate and Fe. Health Canada recently reported that $34 \%$ of adults consume $\mathrm{Mg}$ in quantities below the EAR, with this number being higher than $40 \%$ in some life stage groups. There also is a low prevalence of inadequacy for $\mathrm{Zn}$, folate and $\mathrm{Fe}$, ranging from $10-41 \%$ for specific age and sex groups that consume less than the EAR. Similarly, median $\mathrm{K}$ intakes of Canadian adults are below the adequate intake value ${ }^{(38,39)}$. The current analysis using EAR values was consistent with these findings and further demonstrates that fewer pulse consumers had intakes of nutrients below the EAR.

Pulse consumption is associated with higher $\mathrm{Na}$ intake, contributing to the reported $78 \%$ of Canadian adults that exceed the tolerable upper intake level for $\mathrm{Na}^{(38,39)}$. This is probably not due to the composition of the pulses themselves, but may reflect an increased intake of pulse-containing foods traditionally high in $\mathrm{Na}$, such as Mexican or other Hispanic dishes and bean soups. These dishes account for approximately $25 \%$ of all pulse products consumed. It is also possible that the increased $\mathrm{Na}$ intake among pulse consumers is due to the $\mathrm{Na}$ that is added to canned beans during processing, but such conclusions cannot be made from this dataset. Another potential concern associated with pulse consumption was the lower intakes of vitamins $\mathrm{B}_{12}$ and $\mathrm{D}$, as $10-35 \%$ of Canadian adults have an intake of vitamin $B_{12}$ that is below the $\mathrm{EAR}^{(38,39)}$ and the median intake of vitamin $\mathrm{D}$ in Canada is below the recently revised RDA for this nutrient ${ }^{(38-40,42)}$. However, the proportions of respondents below the EAR for both vitamin $\mathrm{D}$ and $\mathrm{B}_{12}$ were similar among consumers and non-consumers, suggesting that pulse consumption does not compromise the nutrient status of these individuals in this regard and that the majority of Canadians, regardless of pulse consumption, are not consuming sufficient levels of these nutrients.

On the other hand, pulse consumers tended to consume higher levels of $\mathrm{Ca}$, a finding that was significant in the US data analysis. This cannot be explained by altered dairy product intake, as consumption of foods from the milk and milk product group was not increased in pulse consumers. The increase in $\mathrm{Ca}$ is perhaps due to the fact that common pulse dishes such as Hispanic foods contain $\mathrm{Ca}^{(22)}$. Further detailed analysis on the connection between ethnic background and food choices may shed some light on the relationship between pulse intake and vitamin D status.

Overall energy intake was higher in pulse consumers, a finding consistent with the US population analysis ${ }^{(22)}$. It would be expected that this higher energy intake by pulse consumers would be associated with an increased body mass. However, although mean BMI was higher in pulse consumers (28.0 (SE 0.75$)$ and $27.3(\operatorname{se~} 0.11) \mathrm{kg} / \mathrm{m}^{2}$, respectively), this difference was not statistically significant. This trend is in contrast to other findings which suggest that high-fibre foods such as pulses lead to an increased feeling of fullness and may lead to a healthier body weight when eaten at higher amounts ${ }^{(15,16)}$. The reason for this apparent discrepancy cannot be determined from the survey data, but it may be that other foods consumed along with pulses may counteract the expected satiating effects of high-fibre pulses. The effect of pulse consumption on body weight also may be confounded by the fact that a large proportion of Canadians are in the overweight BMI category ${ }^{(43)}$.

In the USA, the percentages of total energy from total and saturated fat were significantly lower in the diets of consumers in the third and fourth quartiles of pulse consumption ${ }^{(22)}$. This trend also was observed in Canadians in the present analysis, but not as strongly as in the US data. It is interesting to note that while US pulse consumers in the highest quartile of intake consume $20 \mathrm{~g}$ more of total fat than non-consumers, Canadians in the third quartile consume $10 \mathrm{~g}$ more total fat, but in the fourth quartile, fat consumption is similar to nonconsumers. These differences may be due to the differences in the types of foods commonly consumed in the different data sets. Mung bean dishes more commonly consumed in Canada are typically lower in fat compared with pinto and refried bean dishes more commonly consumed in the USA. This also may explain why the intakes of MUFA and PUFA in the Canadian pulse consumers were higher than in non-consumers, while this trend was not observed in the US data ${ }^{(22)}$.

In addition to the predominantly positive effect of pulse consumption on nutrient intakes, further evidence of improved dietary quality with pulse consumption is demonstrated by the extra serving of fruits and vegetables eaten by consumers compared with non-consumers, a goal of the Food Guide that most Canadians do not achieve. In contrast, pulse consumers in the NHANES data consumed more grain products, but not other food groups ${ }^{(22)}$. These differences are probably due to the difference in the sources of pulses being eaten by Canadian compared with US consumers.

There are some limitations in this research. As with the NHANES study, the consumption of pulses in the CCHS $2 \cdot 2$ is based on a single reference day, meaning that those who were not identified as a consumer during a single day of intake may still be a pulse consumer. Although second-day consumer values were similar to the first day, the frequency of consumption is impossible to determine based on the $24 \mathrm{~h}$ recall method. Information was not collected on specific types of diets (i.e. low-carbohydrate, vegetarian or vegan diets) which may have been helpful in further examining the demographic of the average consumer, or on food preparation techniques, which may affect the nutrient content (for example, added salt) of the dishes consumed. It is also impossible to determine whether the $24 \mathrm{~h}$ dietary recall was truly representative of the respondent's normal diet, as respondents may over- or under-report their food consumption, even with trained experts administering the dietary recall. Because the CCHS $2 \cdot 2$ was a cross-sectional look at the dietary habits of Canadians, the results should be interpreted with caution. It is entirely possible that the survey would have provided differing results if another time-frame had been chosen, with either higher or lower amounts of consumers. As well, cause and effect cannot be assumed, as pulse consumption may be a component of an overall lifestyle. 
The CCHS 2.2 also does not take into account those residing on Indian reserves, residents occupying any of the three territories, those living in institutions or members of the Canadian Forces $^{(24,25)}$.

These results demonstrate that an increased intake of dry beans and peas, especially at higher intakes, is associated with higher intakes of fibre, protein, carbohydrate, folate, $\mathrm{Mg}, \mathrm{Fe}, \mathrm{K}$ and $\mathrm{Zn}$ in Canadians, leading to improved diet quality. Clarification of the reasons for the effects of pulse consumption on these potential improvements in the diet as well as the potential increase in $\mathrm{Na}$ intake will need to be elucidated so that dietary advice to consume pulses can include ways to enhance or mitigate any positive or negative effects, respectively. In this way, the potential beneficial effects of pulse consumption on nutrient intake can be realised.

\section{Acknowledgements}

We would like to offer many thanks to Dr Ian Clara and Kelly Cranswick at the Manitoba Research Data Centre for their ongoing statistical help and support. The present study was supported by grants from Saskatchewan Pulse Growers and as part of the Pulse Innovation Project, through Canada's Agricultural Policy Framework (APF), a Federal-ProvincialTerritorial initiative. All authors were responsible for the study design and assisted in revision of the manuscript. A. N. M., N. Y. and H. M. A. were responsible for data analyses, interpreting the results, drafting of the manuscript and critical revision of the manuscript. All authors read and approved the final manuscript. All authors declare no conflicts of interest.

\section{References}

1. Leterme P \& Munõz LC (2002) Factors influencing pulse consumption in Latin America. Br J Nutr 88, Suppl. 3, S251-S254.

2. Ofuya ZM \& Akhidue V (2005) The role of pulses in human nutrition: a review. J Appl Sci Environ Mgt 9, 99-104.

3. Food and Agriculture Organization (2010) Crops statistics: concepts, definitions and classifications. http://www. fao.org/economic/ess/methodology/methodology-systems/ crops-statistics-concepts-definitions-and-classifications/en/

4. Food and Agriculture Organization (1994) Definition and classification of commodities: pulses and derived products. http://www.fao.org/es/faodef/fdef04e.htm

5. Michaels TE (2004) Pulses, overview. In Encyclopedia of Grain Science, pp. 494-501 [C Wrigley, H Corke and C Walker, editors]. Kidlington: Elsevier.

6. Patterson CA, Maskus H \& Dupasquier C (2009) Pulse Crops for Health. Pulse Canada: Winnipeg.

7. Health Canada (2008) Investigating the Nutrition and Health Attributes of Beans, Chickpeas, Lentils, Peas: Clinical Trial Research Projects Funded by Canada's Pulse Industry. http://www.has.uwo.ca/hospitality/ibean/resources.cfm

8. Anderson JW, Smith BM \& Washnock CS (1999) Cardiovascular and renal benefits of dry bean and soybean intake. Am J Clin Nutr 70, Suppl. 3, 464S-474S.

9. Tosh S \& Yada S (2010) Dietary fibres in pulse seeds and fractions: characterization, functional attributes, and applications. Food Res Int 43, 450-460.
10. Guenther PM, Dodd KW, Reedy J, et al. (2006) Most Americans eat much less than the recommended amounts of fruits and vegetables. J Am Diet Assoc 106, 1371-1379.

11. Winham D, Webb D \& Barr A (2008) Beans and good health. Nutr Today 43, 201-209.

12. Venter CS \& van Eyssen E (2001) More legumes for better overall health. S Afr J Clin Nutr 14, S32-S38.

13. Messina MJ (2009) Legumes and soybeans: overview of their nutritional profiles and health effects. Am J Clin Nutr 70, 451S-458S.

14. Willet WC, Sacks F, Trichopoulou A, et al. (1995) Mediterranean diet pyramid: a cultural model for healthy eating. $A m J$ Clin Nutr 61, 1402S-1406S.

15. Anderson JW, Smith BM \& Gustafson NJ (1994) Health benefits and practical aspects of high fibre diets. Am J Clin Nutr 59, 1242S-1247S.

16. Papanikolaou Y \& Fulgoni VL (2008) Bean consumption is associated with greater nutrient intake, reduced systolic blood pressure, lower body weight and a smaller waist circumference in adults: Results from the National Health and Nutrition Examination Survey 1999-2002. J Am Coll Nutr 27, 569-576.

17. Jenkins DJA, Axelsen M, Kendall CWC, et al. (2000) Dietary fibre, lentil carbohydrates and the insulin resistant diseases. Br J Nutr 83, Suppl. 1, S157-S163.

18. Rizkalla SW, Bellisle F \& Slama G (2002) Health benefits of low glycaemic index foods, such as pulses, in diabetic patients and healthy individuals. Br J Nutr 88, 255-262.

19. Health Canada (2010) Canada's Food Guide. http://www. hc-sc.gc.ca/fn-an/food-guide-aliment/using-utiliser/countcalcul-eng.php

20. US Department of Health and Human Services (2010) Dietary Guidelines for Americans 2010. http://www.cnpp. usda.gov/dietaryguidelines.htm

21. Putnam J, Kantor LS \& Allshouse J (2000) Per capita food supply trends: progress toward dietary guidelines. Food Rev 23, 2-14.

22. Mitchell DC, Lawrence FR, Hartman TJ, et al. (2009) Consumption of dry beans, peas, and lentils could improve diet quality in the US population. J Am Diet Assoc 109 909-913.

23. Health Canada (2010) Canadian Community Health Survey: Cycle 2-2: a guide to accessing and interpreting the data. http://www.hc-sc.gc.ca/fn-an/surveill/nutrition/commun/cchs_ guide_escc-eng.php

24. Statistics Canada (2010) Canadian Community Health Survey: Cycle 2.2: common questions and qnswers for users. http://www.statcan.gc.ca/imdb-bmdi/document/5049_ D1_T9_V1-eng.pdf

25. Health Canada (2010) Canadian Community Health Survey-Nutrition (CCHS). http://www.statcan.gc.ca/cgiin/ $\mathrm{imdb} / \mathrm{p} 2 S V \cdot$ pl?Function $=$ getSurvey $\&$ SDDS $=5049 \& \mathrm{lang}=\mathrm{en} \&$ $\mathrm{db}=\mathrm{imdb} \& \mathrm{adm}=8 \& \mathrm{dis}=2$

26. Health Canada (2010) Canadian Nutrient File. http://www. hc-sc.gc.ca/fn-an/nutrition/fiche-nutri-data/user_guide_d_ utilisation01-eng.php

27. US Department of Agriculture (2011) Plants Database. http:// plants.usda.gov/java/

28. JJ Otten, J Pitzi Hellwig and LD Meyers (editors) (2006) Dietary Reference Intakes: The Essential Guide to Nutrient Requirements. Washington, DC: National Academies Press.

29. US Department of Agriculture (2010) Nutrient Data Laboratory. http://www.nal.usda.gov/fnic/foodcomp/search/

30. Rao JNK, Wu CFJ \& Yu K (1992) Some recent work on resampling methods for complex surveys. Surv Methodol 18, 209-217. 
31. Rust KF \& Rao JNK (1996) Variance estimation for complex surveys using replication techniques. Stat Methods Med Res 5, 283-310.

32. Health Canada (2009) Canadian Community Health Survey: Detailed Information for 2004 (Cycle 2.2). http://www. statcan.gc.ca/cgi-bin/imdb/p2SV.pl?Function=getSurvey\& SDDS $=5049 \&$ lang $=$ en $\& \mathrm{db}=\mathrm{imdb} \& \mathrm{adm}=8 \& \mathrm{dis}=2$

33. Lucier G, Lin B-H, Allhouse J, et al. (2000) Factors affecting dry bean consumption in the United States. In Vegetables and Specialities Situation and Outlook Report, vol. 280, pp. 26-34. Washington, DC: US Department of Agriculture, Economic Research Service.

34. US Census Bureau (2007) Hispanic Americans by the Numbers. http://www.infoplease.com/spot/hhmcensus1.html

35. Statistics Canada (2006) Visible minority groups, 2006 counts, for Canada, provinces and territories. http://www12.statcan. $\mathrm{ca} /$ census-recensement/2006/dp-pd/hlt/97-562/pages/page. $\mathrm{cfm} ?$ Lang $=\mathrm{E} \& \mathrm{GeO}=\mathrm{PR} \&$ Code $=01 \&$ Table $=1 \&$ Data $=$ Count $\&$ StartRec $=1 \&$ Sort $=2 \&$ Display $=$ Page

36. Canadian Council on Social Development (2004) Demographics of the Canadian Population. http://www.ccsd.ca/ factsheets/demographics/
37. US Census Bureau (2010) Stat and Country Quick Facts. http://quickfacts.census.gov/qfd/states/00000.html

38. Health Canada (2009) Articles on Canadians' Food and Nutrient Intakes - Canadian Community Health Survey, Cycle 2.2, Nutrition (2004). Ottawa: Health Canada.

39. Health Canada (2009) Do Canadian Adults Meet their Nutrient Requirements Through Food Intake Alone? Ottawa: Health Canada.

40. Health Canada (2010) Dietary reference intakes tables. http://www.hc-sc.gc.ca/fn-an/alt_formats/hpfb-dgpsa/pdf/ nutrition/dri_tables-eng.pdf

41. Sgarbieri VC, Antunes PL \& Almeida LD (1979) Nutritional evaluation of four varieties of dry beans. J Food Sci $\mathbf{4 4}$, $1306-1308$.

42. Institute of Medicine of the National Academies (2010) Dietary Reference Intakes for Calcium and Vitamin D. http://www.iom.edu/Reports/2010/Dietary-Reference-Intakesfor-Calcium-and-Vitamin-D.aspx

43. Health Canada (2010) Body Mass Index (BMI) Nomogram. http://www.hc-sc.gc.ca/fn-an/nutrition/weights-poids/guideld-adult/bmi_chart_java-graph_imc_java-eng.php 